\title{
The BCS energy gap at low density
}

\author{
Asbjørn Bækgaard Lauritsen ${ }^{1,2}$ (1)
}

Received: 14 September 2020 / Revised: 4 December 2020 / Accepted: 23 January 2021 /

Published online: 12 February 2021

(c) The Author(s) 2021

\section{Abstract}

We show that the energy gap for the BCS gap equation is

$$
\Xi=\mu\left(8 \mathrm{e}^{-2}+o(1)\right) \exp \left(\frac{\pi}{2 \sqrt{\mu} a}\right)
$$

in the low density limit $\mu \rightarrow 0$. Together with the similar result for the critical temperature by Hainzl and Seiringer (Lett Math Phys 84: 99-107, 2008), this shows that, in the low density limit, the ratio of the energy gap and critical temperature is a universal constant independent of the interaction potential $V$. The results hold for a class of potentials with negative scattering length $a$ and no bound states.

Keywords BCS equation · Energy gap $\cdot$ Superfluidity

Mathematics Subject Classification 81Q10, 46N50, 82D50

\section{Introduction and main result}

The Bardeen-Cooper-Schrieffer (BCS) gap equation at zero temperature

$$
\Delta(p)=-\frac{1}{(2 \pi)^{3 / 2}} \int_{\mathbb{R}^{3}} \hat{V}(p-q) \frac{\Delta(q)}{E(q)} \mathrm{d} q,
$$

where $E(p)=\sqrt{\left(p^{2}-\mu\right)^{2}+|\Delta(p)|^{2}}$, is an important part of the BCS theory of superfluidity and conductivity [1]. The function $\Delta$ has the interpretation of the order parameter describing pairs of Fermions (Cooper pairs). The potential $V$ models an

$\triangle$ Asbjørn Bækgaard Lauritsen

asbjornbaekgaard.lauritsen@ist.ac.at

1 Department of Mathematics, University of Copenhagen, Universitetsparken 5, 2100

Copenhagen, Denmark

2 Present Address: IST Austria (Institute of Science and Technology Austria), Am Campus 1, 3400 Klosterneuburg, Austria 
effective local interaction. (In the case of superconductivity it is between electrons.) We will assume that $V \in L^{1}\left(\mathbb{R}^{3}\right)$, in which case $V$ has a Fourier transform, $\hat{V}(p)=$ $(2 \pi)^{-3 / 2} \int_{\mathbb{R}^{3}} V(x) \mathrm{e}^{-i p x} \mathrm{~d} x$. Under the assumption $\hat{V} \leq 0$, it is proved in [7] that a solution $\Delta$ to the BCS gap equation is unique (up to a constant global phase).

We will here study the low density limit, $\mu \rightarrow 0$, of the energy gap (at zero temperature)

$$
\Xi=\inf E(p)=\inf \sqrt{\left(p^{2}-\mu\right)^{2}+|\Delta(p)|^{2}} .
$$

The function $E$ has the interpretation as the dispersion relation for the corresponding BCS Hamiltonian, and so $\Xi$ is indeed an energy gap, see [5, Appendix A]. This limit has previously been studied in [6], where the critical temperature has been calculated, and it is known that, in this limit, superfluid/conducting behaviour is well described by BCS theory $[10,12]$. The critical temperature is another key feature of BCS theory. For temperatures below the critical temperature, the system is in a superfluid/conducting state. For temperatures above, it is not.

BCS theory has also been studied in the weak coupling limit [7], where one considers a potential $\lambda V$ for a fixed $V$ in the limit of a small coupling constant $\lambda \rightarrow 0$. In that limit, it is shown that the energy gap satisfies $\Xi \sim A \exp (-B / \lambda)$ for explicit constants $A, B>0$.

In the low density limit, it turns out that the energy gap, as the critical temperature [6], is related to the scattering length of the potential $2 \mathrm{~V}$, which we now define.

Definition 1 [6, Definition 2] Let $V \in L^{1}\left(\mathbb{R}^{3}\right) \cap L^{3 / 2}\left(\mathbb{R}^{3}\right)$ be real-valued. By $V(x)^{1 / 2}$, we will mean $V(x)^{1 / 2}=\operatorname{sgn}(V(x))|V(x)|^{1 / 2}$. Suppose that -1 is not in the spectrum of the associated Birman-Schwinger operator $V^{1 / 2} \frac{1}{p^{2}}|V|^{1 / 2}$. Then, the scattering length $a$ of $2 V$ is

$$
a=\frac{1}{4 \pi}\left\langle|V|^{1 / 2}\left|\frac{1}{1+V^{1 / 2} \frac{1}{p^{2}}|V|^{1 / 2}}\right| V^{1 / 2}\right\rangle .
$$

Here, operators that are functions of $p$ are to be interpreted as multiplication operators in Fourier space.

In [6, Appendix A] it is explained, why it is sensible to call this a scattering length. With this, we may now state our main theorem.

Theorem 1 Let $V$ be radial and assume that $V(x)(1+|x|) \in L^{1}\left(\mathbb{R}^{3}\right) \cap L^{3 / 2}\left(\mathbb{R}^{3}\right), \hat{V} \leq 0$, $\hat{V}(0)<0$, that $\|V\|_{L^{3 / 2}}<S_{3}$, and that the scattering length $a<0$ is negative. Then,

$$
\lim _{\mu \rightarrow 0}\left(\log \frac{\mu}{\Xi}+\frac{\pi}{2 \sqrt{\mu} a}\right)=2-\log 8 .
$$

That is, in the limit of low density, the energy gap satisfies

$$
\Xi=\mu\left(8 \mathrm{e}^{-2}+o(1)\right) \exp \left(\frac{\pi}{2 \sqrt{\mu} a}\right) .
$$


This is known in the physics literature [10]. Here, $S_{3}=\frac{3}{4} 2^{2 / 3} \pi^{4 / 3} \approx 5.4779$ is the best constant in Sobolev's inequality [11, Theorem 8.3]. The assumption that $\|V\|_{L^{3 / 2}}<S_{3}$ gives that $p^{2}+\lambda V>0$ for any $0<\lambda \leq 1$ by Sobolev's inequality, see [11, section 11.3]. Thus, by the Birman-Schwinger principle, the operator $\lambda V^{1 / 2} \frac{1}{p^{2}}|V|^{1 / 2}$ does not have -1 as an eigenvalue. Varying $\lambda$ we thus get that the spectrum of $V^{1 / 2} \frac{1}{p^{2}}|V|^{1 / 2}$ is contained in $(-1, \infty)$. In particular, the scattering length is finite. Also, for a $V$ satisfying the assumptions it also satisfies the assumptions of [6, Theorem 1]. This states that the critical temperature satisfies

$$
T_{c}=\mu\left(\frac{8}{\pi} \mathrm{e}^{\gamma-2}+o(1)\right) \exp \left(\frac{\pi}{2 \sqrt{\mu} a}\right)
$$

where $\gamma \approx 0.577$ is the Euler-Mascheroni constant. We thus immediately get the following.

Corollary 1 Let $V$ be radial and assume that $V(x)(1+|x|) \in L^{1}\left(\mathbb{R}^{3}\right) \cap L^{3 / 2}\left(\mathbb{R}^{3}\right)$, $\hat{V} \leq 0, \hat{V}(0)<0$, that $\|V\|_{L^{3 / 2}}<S_{3}$, and that the scattering length $a<0$ is negative. Then,

$$
\lim _{\mu \rightarrow 0} \frac{\Xi}{T_{c}}=\pi \mathrm{e}^{-\gamma} \approx 1.7639
$$

That is, in the low density limit, the ratio of the energy gap and critical temperature tends to some universal limit independent of the potential $V$. This is known in the physics literature [4]. Also, this property has been observed before in the weak coupling limit $[1,7,12]$. In [7], by considering the potential $\lambda V$ for $V$ fixed, it is shown that $\Xi / T_{c} \rightarrow \pi \mathrm{e}^{-\gamma}$ when $\lambda \rightarrow 0$. That is, there exists such universal constants in both the low density and weak coupling limits, and moreover, they are the same in both limits.

The assumptions we impose on the potential $V$ is more or less the assumptions of $[6,7]$. The only difference is the assumption that $\|V\|_{L^{3 / 2}}<S_{3}$ instead of the assumption that $V^{1 / 2} \frac{1}{p^{2}}|V|^{1 / 2}$ has spectrum contained in $(-1, \infty)$. As discussed above, our assumption here is stronger. We need such a stronger assumption, since we need to control different scalings of the potential. As discussed in [6] our assumption captures that the operator $p^{2}+V$ does not have any bound states.

We will follow the description of BCS theory made in [2,3,5-9]. There, the BCS gap equation at zero temperature arises as the Euler-Lagrange equations for minimisers of the BCS functional at zero temperature

$$
\mathcal{F}^{\mu, V}(\alpha)=\frac{1}{2} \int\left|p^{2}-\mu\right|\left(1-\sqrt{1-4|\hat{\alpha}(p)|^{2}}\right) \mathrm{d} p+\int V(x)|\alpha(x)|^{2} \mathrm{~d} x .
$$

For a minimiser $\alpha$ one then defines

$$
\Delta(p)=-2 \widehat{V \alpha}(p)
$$

This $\Delta$ then satisfies the BCS gap equation, see [9]. 
The BCS functional satisfies the scaling

$$
\begin{aligned}
& \mathcal{F}^{\mu, V}(\alpha)=\mu^{5 / 2} \mathcal{F}^{1, \sqrt{\mu} V_{\sqrt{\mu}}(\beta)} \\
& \quad=\mu^{5 / 2}\left[\frac{1}{2} \int\left|p^{2}-1\right|\left(1-\sqrt{1-4|\hat{\beta}(p)|^{2}}\right) \mathrm{d} p+\sqrt{\mu} \int V_{\sqrt{\mu}}|\beta|^{2} \mathrm{~d} x\right],
\end{aligned}
$$

where $\beta(x)=\mu^{-3 / 2} \alpha(x / \sqrt{\mu})$ and $V_{\sqrt{\mu}}(x)=\mu^{-3 / 2} V(x / \sqrt{\mu})$. If we replaced the potential $V_{\sqrt{\mu}}$ by some constant potential, this would correspond to the weak coupling limit, studied in [7] (with coupling constant $\lambda=\sqrt{\mu}$ ). The potential $V_{\sqrt{\mu}}$ is of course not constant, so the methods and results of [7] do not (immediately) apply.

We will not pursue the idea of trying to extend the results of [7] to our case. Instead, we will use the methods of [6]. This approach has the advantage that the asymptotic of $\Xi$ comes out in a form, where the scattering length $a$ appears explicitly, and thus allows us to easily compare this asymptotic to that of $T_{c}$ from [6], thus giving us Corollary 1.

We now give the proof of our theorem. The first part is novel and consists of getting a priori bounds on the minimiser $\alpha$ of the BCS functional and the corresponding solution to the BCS gap equation $\Delta$ that are good enough for us to apply the methods of [6]. The second part applies the methods of [6] to our case.

\section{Proof}

One of the key ideas in the proof is to study the asymptotic of $m_{\mu}(\Delta)=\frac{1}{(2 \pi)^{3}} \int \frac{1}{E(p)}-\frac{1}{p^{2}} \mathrm{~d} p=\frac{1}{(2 \pi)^{3}} \int \frac{1}{\sqrt{\left|p^{2}-\mu\right|^{2}+|\Delta(p)|^{2}}}-\frac{1}{p^{2}} \mathrm{~d} p$.

This is similar to what is done in [6,7] for the study of the critical temperature and energy gap in the weak coupling limit and for the critical temperature in the low density limit.

In [7, Lemma 2], it is proven that there exists a unique minimiser $\alpha$ of the BCS functional at zero temperature with (strictly) positive Fourier transform. This we will denote by $\alpha_{\mu, V}$. Since the BCS functional is invariant under rotation, it follows that $\alpha_{\mu, V}$ and thus also $\Delta=-2 \widehat{V \alpha_{\mu, V}}$ are radial functions [7]. Additionally, since $\hat{V} \leq 0$ we have that $\Delta \geq 0$. By the BCS equation, it follows that even $\Delta>0$, see [7].

By the scaling of the BCS functional, Eq. (1), we see that $\alpha_{\mu, V}$ satisfies the scaling

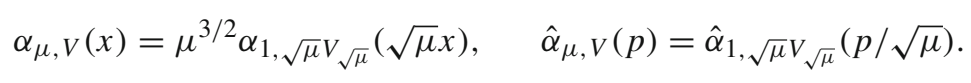

The asymptotics of $m_{\mu}(\Delta)$ and $\Xi$ are as follows.

Lemma 1 In the limit $\mu \rightarrow 0$, we have

- $m_{\mu}(\Delta)=\frac{\sqrt{\mu}}{2 \pi^{2}}\left(\log \frac{\mu}{\Delta(\sqrt{\mu})}-2+\log 8+o(1)\right)$ 
- $m_{\mu}(\Delta)=\frac{-1}{4 \pi a}+o\left(\mu^{1 / 2}\right)$

- $\Xi=\Delta(\sqrt{\mu})(1+o(1))$.

By $\Delta(\sqrt{\mu})$, we mean the value of $\Delta$ on a sphere of radius $\sqrt{\mu}$. Since $\Delta$ is radial, this is well defined. The first equality here may be reformulated as $\Delta(\sqrt{\mu})=$ $\mu\left(8 \mathrm{e}^{-2}+o(1)\right) \exp \left(-\frac{2 \pi^{2} m_{\mu}(\Delta)}{\sqrt{\mu}}\right)$.

With this, we may prove our main theorem.

Proof (of theorem 1) By Lemma 1, we get

$$
\begin{aligned}
\lim _{\mu \rightarrow 0}\left(\log \frac{\mu}{\Xi}+\frac{\pi}{2 \sqrt{\mu} a}\right) & =\lim _{\mu \rightarrow 0}\left(\log \frac{\mu}{\Delta(\sqrt{\mu})}+\frac{\pi}{2 \sqrt{\mu} a}\right) \\
& =\lim _{\mu \rightarrow 0}\left(\log \frac{\mu}{\Delta(\sqrt{\mu})}-\frac{2 \pi^{2} m_{\mu}(\Delta)}{\sqrt{\mu}}\right)=2-\log 8
\end{aligned}
$$

This concludes the proof of Theorem 1 .

We now give the proof of Lemma 1. The structure of the proof is as follows. First, we find bounds on the minimiser $\alpha$ of the BCS functional. These then translate to bounds on the function $\Delta$, which gives some asymptotic behaviour of $m_{\mu}(\Delta)$. Armed with this, we employ the methods of [6] to improve on these a priori results.

Proposition 1 In the limit $\mu \rightarrow 0$, the minimiser satisfies $\left\|\alpha_{\mu, V}\right\|_{H^{1}} \leq C \mu^{3 / 4}$.

Proof By the scaling of $\alpha_{\mu, V}$ we compute (for $\mu \leq 1$ )

$$
\begin{aligned}
& \left\|\alpha_{\mu, V}\right\|_{H^{1}}^{2}=\int\left|\hat{\alpha}_{\mu, V}(p)\right|^{2}\left(1+p^{2}\right) \mathrm{d} p
\end{aligned}
$$

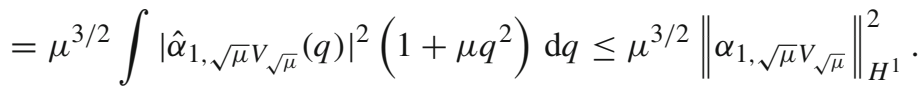

We now show, that this latter norm is bounded uniformly in $\mu$.

Let $\lambda=\frac{S_{3}}{\|V\|_{L^{3 / 2}}}>1$. Then, as $\left\|\sqrt{\mu} V_{\sqrt{\mu}}\right\|_{L^{3 / 2}}=\|V\|_{L^{3 / 2}}$ it follows that $\frac{p^{2}}{\lambda}+\sqrt{\mu} V_{\sqrt{\mu}} \geq 0$ by Sobolev's inequality, see [11, section. 11.3]. Using that $1-\sqrt{1-4 x^{2}} \geq 2 x^{2}$, we may bound for any $\alpha$,

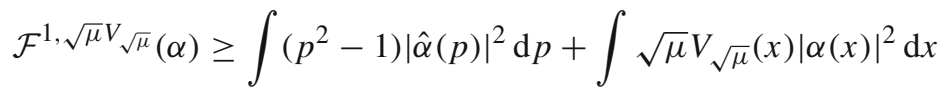

$$
\begin{aligned}
& =\left\langle\alpha\left|\frac{p^{2}}{\lambda}+\sqrt{\mu} V_{\sqrt{\mu}}\right| \alpha\right\rangle+\int\left(2 \varepsilon p^{2}-1\right)|\hat{\alpha}(p)|^{2} \mathrm{~d} p \\
& \geq \varepsilon \int|\hat{\alpha}(p)|^{2}\left(1+p^{2}\right) \mathrm{d} p+\int\left(\varepsilon p^{2}-\varepsilon-1\right)|\hat{\alpha}(p)|^{2} \mathrm{~d} p \\
& \geq \varepsilon\|\alpha\|_{H^{1}}^{2}-A \text {, }
\end{aligned}
$$


where we introduced $\varepsilon=\frac{1}{2}-\frac{1}{2 \lambda}>0$ and $A=\frac{1}{4} \int\left[\varepsilon p^{2}-1-\varepsilon\right]_{-} \mathrm{d} p<\infty$. Since

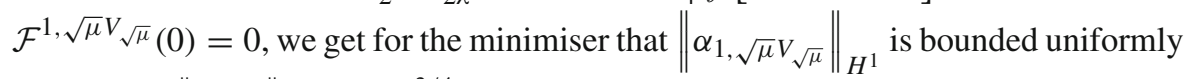
in $\mu$. Thus, $\left\|\alpha_{\mu, V}\right\|_{H^{1}} \leq C \mu^{3 / 4}$ for small $\mu$.

Proposition 2 For small enough $\mu$, the minimiser satisfies

$$
\left\|\hat{\alpha}_{\mu, V} 1_{\{|p|>\varepsilon\}}\right\|_{L^{3 / 2}} \leq C\left\|\hat{\alpha}_{\mu, V} 1_{\{|p| \leq \varepsilon\}}\right\|_{L^{1}}
$$

for a small $\varepsilon>0$ and a constant $C$, both independent of $\mu$.

Proof By the continuity of $\hat{V}$, we may find $\varepsilon>0$ such that $2 \hat{V}(0) \leq \hat{V}(p) \leq$ $\frac{1}{2} \hat{V}(0)<0$ for all $|p| \leq 2 \varepsilon$. Let $\lambda=\frac{S_{3}}{\|V\|_{L^{3 / 2}}}>1$. Then, $\frac{p^{2}}{\lambda}+V \geq 0$. For the minimiser $\alpha=\alpha_{\mu, V}$, we have again using the inequality $1-\sqrt{1-4 x^{2}} \geq 2 x^{2}$, the following.

$$
\begin{aligned}
\mathcal{F}^{\mu, V} & (\alpha)=\frac{1}{2} \int\left|p^{2}-\mu\right|\left(1-\sqrt{1-4 \hat{\alpha}(p)^{2}}\right) \mathrm{d} p+\int V(x)|\alpha(x)|^{2} \mathrm{~d} x \\
= & \frac{1}{2} \int_{|p|>\varepsilon}\left|p^{2}-\mu\right|\left(1-\sqrt{1-4 \hat{\alpha}(p)^{2}}\right) \mathrm{d} p \\
& +\frac{1}{2} \int_{|p| \leq \varepsilon}\left|p^{2}-\mu\right|\left(1-\sqrt{1-4 \hat{\alpha}(p)^{2}}\right) \mathrm{d} p \\
& +\frac{1}{(2 \pi)^{3 / 2}} \iint \hat{\alpha}(p) \hat{V}(p-q) \hat{\alpha}(q) \mathrm{d} p \mathrm{~d} q \\
\geq & \int_{|p|>\varepsilon}\left(p^{2}-\mu\right) \hat{\alpha}(p)^{2} \mathrm{~d} p+\frac{1}{(2 \pi)^{3 / 2}} \iint_{\hat{\alpha}}(p) \hat{V}(p-q) \hat{\alpha}(q) \mathrm{d} p \mathrm{~d} q \\
= & \int_{|p|>\varepsilon}\left(p^{2}-\mu\right) \hat{\alpha}(p)^{2} \mathrm{~d} p+\frac{1}{(2 \pi)^{3 / 2}}\left[\int_{|p| \leq \varepsilon} \int_{|q| \leq \varepsilon} \hat{\alpha}(p) \hat{V}(p-q) \hat{\alpha}(q) \mathrm{d} p \mathrm{~d} q\right. \\
& +2 \int_{|p| \leq \varepsilon \mid} \int_{|q|>\varepsilon} \hat{\alpha}(p) \hat{V}(p-q) \hat{\alpha}(q) \mathrm{d} p \mathrm{~d} q \\
& \left.+\int_{|p|>\varepsilon \mid} \int_{|q|>\varepsilon} \hat{\alpha}(p) \hat{V}(p-q) \hat{\alpha}(q) \mathrm{d} p \mathrm{~d} q\right] \\
\geq & \left\langle\hat{\alpha} 1_{\{|p|>\varepsilon\}}\left|\frac{p^{2}}{\lambda}+V\right| \hat{\alpha} 1_{\{|p|>\varepsilon\}}\right\rangle+\int_{|p|>\varepsilon}\left(\left(1-\frac{1}{\lambda}\right) p^{2}-\mu\right) \hat{\alpha}(p)^{2} \mathrm{~d} p \\
& +\frac{1}{(2 \pi)^{3 / 2}}\left[2 \hat{V}(0)\left\|\hat{\alpha} 1_{\{|p| \leq \varepsilon\}}\right\|_{L^{1}}^{2}+2 \int_{|p| \leq \varepsilon} \int_{|q|>\varepsilon} \hat{\alpha}(p) \hat{V}(p-q) \hat{\alpha}(q) \mathrm{d} p \mathrm{~d} q\right] \\
\geq & \int_{|p|>\varepsilon}\left(\left(1-\frac{1}{\lambda}\right) p^{2}-\mu\right) \hat{\alpha}(p)^{2} \mathrm{~d} p \\
& +\frac{1}{(2 \pi)^{3 / 2}}\left[2 \hat{V}(0)\left\|\hat{\alpha} 1_{\{|p| \leq \varepsilon\}}\right\|_{L^{1}}^{2}+2 \int_{|p| \leq \varepsilon} \int_{|q|>\varepsilon} \hat{\alpha}(p) \hat{V}(p-q) \hat{\alpha}(q) \mathrm{d} p \mathrm{~d} q\right] .
\end{aligned}
$$


We now bound the two remaining integrals. For the first integral, we have for sufficiently small $\mu$ that

$$
\begin{aligned}
& \int_{|p|>\varepsilon}\left(\left(1-\frac{1}{\lambda}\right) p^{2}-\mu\right) \hat{\alpha}(p)^{2} \mathrm{~d} p \\
& \geq c \int_{|p|>\varepsilon} \hat{\alpha}(p)^{2}\left(1+p^{2}\right) \mathrm{d} p \geq c\left\|\hat{\alpha} 1_{\{|p|>\varepsilon\}}\right\|_{L^{3 / 2}}^{2},
\end{aligned}
$$

by the bound $\|\hat{g}\|_{L^{3 / 2}} \leq C\|g\|_{H^{1}}$, valid for any function $g$. To see this, simply write

$$
\begin{aligned}
\|\hat{g}\|_{L^{3 / 2}}^{3 / 2} & =\int|\hat{g}(p)|^{3 / 2} \frac{\left(1+p^{2}\right)^{3 / 4}}{\left(1+p^{2}\right)^{3 / 4}} \mathrm{~d} p \\
& \leq\left(\int|\hat{g}(p)|^{2}\left(1+p^{2}\right) \mathrm{d} p\right)^{3 / 4}\left(\int \frac{1}{\left(1+p^{2}\right)^{3}} \mathrm{~d} p\right)^{1 / 4}=C\|g\|_{H^{1}}^{3 / 2}
\end{aligned}
$$

For the double integral, we use the Young and the Hausdorff-Young inequalities [11, Theorems 4.2 and 5.7]. This gives

$$
\begin{aligned}
\left|\int_{|p| \leq \varepsilon} \int_{|q|>\varepsilon} \hat{\alpha}(p) \hat{V}(p-q) \hat{\alpha}(q) \mathrm{d} p \mathrm{~d} q\right| & \leq C\left\|\hat{\alpha} 1_{\{|p| \leq \varepsilon\}}\right\|_{L^{1}}\|\hat{V}\|_{L^{3}}\left\|\hat{\alpha} 1_{\{|p|>\varepsilon\}}\right\|_{L^{3 / 2}} \\
& \leq C\|V\|_{L^{3 / 2}}\left\|\hat{\alpha} 1_{\{|p| \leq \varepsilon\}}\right\|_{L^{1}}\left\|\hat{\alpha} 1_{\{|p|>\varepsilon\}}\right\|_{L^{3 / 2}} .
\end{aligned}
$$

Combining all this, we get the bound

$$
\begin{aligned}
\mathcal{F}^{\mu, V}(\alpha) \geq & c\left\|\hat{\alpha} 1_{\{|p|>\varepsilon\}}\right\|_{L^{3 / 2}}^{2}-C_{1}\left\|\hat{\alpha} 1_{\{|p|>\varepsilon\}}\right\|_{L^{3 / 2}}\left\|\hat{\alpha} 1_{\{|p| \leq \varepsilon\}}\right\|_{L^{1}} \\
& -C_{2}\left\|\hat{\alpha} 1_{\{|p| \leq \varepsilon\}}\right\|_{L^{1}}^{2},
\end{aligned}
$$

where we absorbed the factors of $V$ into the constants $C_{1}, C_{2}>0$. The right hand side above is a second-degree polynomial in $\left\|\hat{\alpha} 1_{\{|p|>\varepsilon\}}\right\|_{L^{3 / 2}}$. Moreover, the minimiser $\alpha=\alpha_{\mu, V}$ satisfies $\mathcal{F}^{\mu, V}(\alpha) \leq 0$. We conclude that $\left\|\hat{\alpha} 1_{\{|p|>\varepsilon\}}\right\|_{L^{3 / 2}}$ is between the two roots of the second-degree polynomial. In particular,

$$
\begin{aligned}
\left\|\hat{\alpha} 1_{\{|p|>\varepsilon\}}\right\|_{L^{3 / 2}} & \leq \frac{C_{1}\left\|\hat{\alpha} 1_{\{|p| \leq \varepsilon\}}\right\|_{L^{1}}+\sqrt{C_{1}^{2}\left\|\hat{\alpha} 1_{\{|p| \leq \varepsilon\}}\right\|_{L^{1}}^{2}+4 c C_{2}\left\|\hat{\alpha} 1_{\{|p| \leq \varepsilon\}}\right\|_{L^{1}}^{2}}}{2 c} \\
& \leq C\left\|\hat{\alpha} 1_{\{|p| \leq \varepsilon\}}\right\|_{L^{1}}
\end{aligned}
$$

as desired.

We now bound $\Delta=-2 \widehat{V \alpha_{\mu, V}}=-2(2 \pi)^{-3 / 2} \hat{V} * \hat{\alpha}_{\mu, V}$.

Proposition 3 The function $\Delta$ satisfies

$$
\Delta(p) \leq C \mu^{3 / 4} \quad \text { and } \quad\left|\Delta\left(p^{\prime}\right)-\Delta(p)\right| \leq C \mu^{3 / 4}\left|p^{\prime}-p\right| .
$$


Proof We compute

$$
\begin{aligned}
\Delta(p) & =\frac{2}{(2 \pi)^{3 / 2}} \int|\hat{V}(p-q)| \hat{\alpha}_{\mu, V}(q) \mathrm{d} q \\
& \leq C\|\hat{V}\|_{L^{3}}\left\|\hat{\alpha}_{\mu, V}\right\|_{L^{3 / 2}} \leq C\|V\|_{L^{3 / 2}}\left\|\alpha_{\mu, V}\right\|_{H^{1}} \leq C \mu^{3 / 4}
\end{aligned}
$$

by the Hausdorff-Young inequality [11, Theorem 5.7] and the fact that $\|\hat{g}\|_{L^{3 / 2}} \leq$ $C\|g\|_{H^{1}}$. The bound for the difference is similar, using that

$$
\begin{aligned}
\left\|\hat{V}\left(p^{\prime}-\cdot\right)-\hat{V}(p-\cdot)\right\|_{L^{3}} & \leq C\left(\int\left|\mathrm{e}^{-i p^{\prime} x}-\mathrm{e}^{-i p x}\right|^{3 / 2}|V(x)|^{3 / 2} \mathrm{~d} x\right)^{2 / 3} \\
& \leq C\left(\int\left|p^{\prime}-p\right|^{3 / 2}|x|^{3 / 2}|V(x)|^{3 / 2} \mathrm{~d} x\right)^{2 / 3} \\
& =C\|V|\cdot|\|_{L^{3 / 2}}\left|p^{\prime}-p\right|,
\end{aligned}
$$

since $\hat{V}\left(p^{\prime}-\cdot\right)-\hat{V}(p-\cdot)$ is the Fourier transform of $\left(\mathrm{e}^{-i p^{\prime} x}-\mathrm{e}^{-i p x}\right) V(-x)$.

With this bound on $\Delta$, we may prove the third equality in Lemma 1 , i.e. that $\Xi=$ $\Delta(\sqrt{\mu})(1+o(1))$, as follows.

Clearly, $\Xi \leq \Delta(\sqrt{\mu})$. On the other hand, for $\left|p^{2}-\mu\right| \leq \Xi \leq \Delta(\sqrt{\mu})$ we have

$$
|\Delta(p)-\Delta(\sqrt{\mu})| \leq C \mu^{3 / 4}|| p|-\sqrt{\mu}| \leq C \mu^{3 / 4} \frac{\Delta(\sqrt{\mu})}{|p|+\sqrt{\mu}} \leq C \mu^{1 / 4} \Delta(\sqrt{\mu})
$$

and so $\Xi \geq \min _{\left|p^{2}-\mu\right| \leq \Xi} \Delta(p) \geq \Delta(\sqrt{\mu})(1+o(1))$. We conclude that $\Xi=$ $\Delta(\sqrt{\mu})(1+o(1))$.

We now use this bound on $\Delta$ to get some control over $m_{\mu}(\Delta)$. By computing the spherical part of the integral, splitting the integral according to $p^{2}<2 \mu$ and $p^{2}>2 \mu$, and using the substitutions $s=\frac{\mu-p^{2}}{\mu}$ and $s=\frac{p^{2}-\mu}{\mu}$, we may rewrite $m_{\mu}(\Delta)$ as

$$
\begin{aligned}
& m_{\mu}(\Delta)=\frac{\sqrt{\mu}}{4 \pi^{2}}\left[\int_{0}^{1} \frac{\sqrt{1-s}-1}{\sqrt{s^{2}+\left(\frac{\Delta(\sqrt{\mu} \sqrt{1-s})}{\mu}\right)^{2}}}+\frac{\sqrt{1+s}-1}{\sqrt{s^{2}+\left(\frac{\Delta(\sqrt{\mu} \sqrt{1+s})}{\mu}\right)^{2}}}\right. \\
& -\frac{1}{\sqrt{1-s}}-\frac{1}{\sqrt{1+s}} \mathrm{~d} s \\
& +\int_{0}^{1} \frac{1}{\sqrt{s^{2}+\left(\frac{\Delta(\sqrt{\mu} \sqrt{1-s})}{\mu}\right)^{2}}}+\frac{1}{\sqrt{s^{2}+\left(\frac{\Delta(\sqrt{\mu} \sqrt{1+s})}{\mu}\right)^{2}}} \mathrm{~d} s
\end{aligned}
$$




$$
\left.+\int_{1}^{\infty} \frac{\sqrt{1+s}}{\sqrt{s^{2}+\left(\frac{\Delta(\sqrt{\mu} \sqrt{1+s})}{\mu}\right)^{2}}}-\frac{1}{\sqrt{1+s}} \mathrm{~d} s\right] .
$$

Here, by $\Delta(\sqrt{\mu} \sqrt{1 \pm s})$ we (again) mean the value of $\Delta$ on a sphere with the given radius. Since $\Delta$ is radial, this is well defined. We now claim that

Proposition 4 In the limit $\mu \rightarrow 0$, the value $m_{\mu}(\Delta)$ satisfies

$$
\begin{aligned}
& m_{\mu}(\Delta)=\frac{\sqrt{\mu}}{4 \pi^{2}}\left[\int_{0}^{1} \frac{\sqrt{1-s}-1}{\sqrt{s^{2}+\left(\frac{\Delta(\sqrt{\mu})}{\mu}\right)^{2}}}+\frac{\sqrt{1+s}-1}{\sqrt{s^{2}+\left(\frac{\Delta(\sqrt{\mu})}{\mu}\right)^{2}}}-\frac{1}{\sqrt{1-s}}-\frac{1}{\sqrt{1+s}} d s\right. \\
& \left.+\int_{0}^{1} \frac{2}{\sqrt{s^{2}+\left(\frac{\Delta(\sqrt{\mu})}{\mu}\right)^{2}}} d s+\int_{1}^{\infty} \frac{\sqrt{1+s}}{\sqrt{s^{2}+\left(\frac{\Delta(\sqrt{\mu})}{\mu}\right)^{2}}}-\frac{1}{\sqrt{1+s}} d s+o(1)\right] .
\end{aligned}
$$

Proof For the first and last integrals, this follows by a dominated convergence argument. One considers the difference between the claimed value and the known value and uses a dominated convergence argument to shows that this vanishes. For the middle integral, we use Propositions 2 and 3. The argument is as follows.

Define the function(s) $x(s)=\frac{\Delta(\sqrt{1 \pm s} \sqrt{\mu})}{\mu}$. We must then show that

$$
\lim _{\mu \rightarrow 0} \int_{0}^{1} \frac{1}{\sqrt{s^{2}+x(s)^{2}}}-\frac{1}{\sqrt{s^{2}+x(0)^{2}}} \mathrm{~d} s=0 .
$$

First, the function $\Delta$ satisfies (with $\varepsilon>0$ chosen from Proposition 2)

$$
\begin{aligned}
\Delta(p)= & \frac{2}{(2 \pi)^{3 / 2}} \int|\hat{V}(p-q)| \hat{\alpha}_{\mu, V}(q) \mathrm{d} q \\
= & \frac{2}{(2 \pi)^{3 / 2}} \int_{|q| \leq \varepsilon}|\hat{V}(p-q)| \hat{\alpha}_{\mu, V}(q) \mathrm{d} q \\
& \quad+\frac{2}{(2 \pi)^{3 / 2}} \int_{|q|>\varepsilon}|\hat{V}(p-q)| \hat{\alpha}_{\mu, V}(q) \mathrm{d} q .
\end{aligned}
$$

This gives for $|p|=\sqrt{\mu}$ that

$$
\begin{aligned}
\Delta(\sqrt{\mu})= & \frac{2}{(2 \pi)^{3 / 2}} \int_{|q| \leq \varepsilon}|\hat{V}(p-q)| \hat{\alpha}_{\mu, V}(q) \mathrm{d} q \\
& +\frac{2}{(2 \pi)^{3 / 2}} \int_{|q|>\varepsilon}|\hat{V}(p-q)| \hat{\alpha}_{\mu, V}(q) \mathrm{d} q \\
\geq & \frac{1}{(2 \pi)^{3 / 2}}|\hat{V}(0)|\left\|\hat{\alpha}_{\mu, V} 1_{\{|p| \leq \varepsilon\}}\right\|_{L^{1}} .
\end{aligned}
$$


Also, for any $|p|=\sqrt{1 \pm s} \sqrt{\mu}$ that

$$
\begin{aligned}
\Delta(p)= & \frac{2}{(2 \pi)^{3 / 2}} \int_{|q| \leq \varepsilon}|\hat{V}(p-q)| \hat{\alpha}_{\mu, V}(q) \mathrm{d} q \\
& +\frac{2}{(2 \pi)^{3 / 2}} \int_{|q|>\varepsilon}|\hat{V}(p-q)| \hat{\alpha}_{\mu, V}(q) \mathrm{d} q \\
\leq & \frac{4}{(2 \pi)^{3 / 2}}|\hat{V}(0)|\left\|\hat{\alpha}_{\mu, V} 1_{\{|p| \leq \varepsilon\}}\right\|_{L^{1}}+\frac{2}{(2 \pi)^{3 / 2}}\|\hat{V}\|_{L^{3}}\left\|\hat{\alpha}_{\mu, V} 1_{\{|p|>\varepsilon\}}\right\|_{L^{3 / 2}} \\
\leq & C\left\|\hat{\alpha}_{\mu, V} 1_{\{|p| \leq \varepsilon\}}\right\|_{L^{1}} \leq C \Delta(\sqrt{\mu}),
\end{aligned}
$$

by the Hausdorff-Young inequality [11, Theorem 5.7] and Proposition 2. Thus, the function(s) $x(s)$ satisfies $x(s) \leq C x(0)$. With this, we may now prove the desired convergence of integrals.

$$
\begin{aligned}
& \left|\frac{1}{\sqrt{s^{2}+x(s)^{2}}}-\frac{1}{\sqrt{s^{2}+x(0)^{2}}}\right| \\
& =\frac{\left|x(s)^{2}-x(0)^{2}\right|}{\sqrt{s^{2}+x(s)^{2}} \sqrt{s^{2}+x(0)^{2}}\left(\sqrt{s^{2}+x(s)^{2}}+\sqrt{s^{2}+x(0)^{2}}\right)} \\
& \leq \frac{C \mu^{1 / 4} s x(0)}{\sqrt{s^{2}+x(s)^{2}} \sqrt{s^{2}+x(0)^{2}}\left(s+\sqrt{s^{2}+x(0)^{2}}\right)} \\
& \leq C \mu^{1 / 4} \frac{x(0)}{\sqrt{s^{2}+x(0)^{2}}\left(s+\sqrt{s^{2}+x(0)^{2}}\right)}
\end{aligned}
$$

since $|x(s)-x(0)| \leq C \mu^{1 / 4} s$ by Proposition 3 . Now, one may compute that

$$
\int_{0}^{1} \frac{x(0)}{\sqrt{s^{2}+x(0)^{2}}\left(s+\sqrt{s^{2}+x(0)^{2}}\right)} \mathrm{d} s=O(1) .
$$

This shows that

$$
\int_{0}^{1} \frac{1}{\sqrt{s^{2}+x(s)^{2}}}-\frac{1}{\sqrt{s^{2}+x(0)^{2}}} \mathrm{~d} s=O\left(\mu^{1 / 4}\right)
$$

vanishes as desired. We conclude the desired.

The remainder of this paper uses the methods of [6]. We decompose

$$
B_{\Delta}:=V^{1 / 2} \frac{1}{E}|V|^{1 / 2}=V^{1 / 2} \frac{1}{p^{2}}|V|^{1 / 2}+m_{\mu}(\Delta)\left|V^{1 / 2}\right\rangle\left\langle|V|^{1 / 2}\right|+A_{\Delta, \mu},
$$


where $A_{\Delta, \mu}$ is defined such that this holds. That is, its kernel is

$$
A_{\Delta, \mu}(x, y)=V(x)^{1 / 2}|V(y)|^{1 / 2} \frac{1}{2 \pi^{2}} \int_{0}^{\infty}\left(\frac{\sin p|x-y|}{p|x-y|}-1\right)\left(\frac{1}{E}-\frac{1}{p^{2}}\right) p^{2} \mathrm{~d} p
$$

In order to see this, note that $\int_{S^{2}} \mathrm{e}^{i p x} \mathrm{~d} p=4 \pi \frac{\sin |x|}{|x|}$. The operator $B_{\Delta}$ is the BirmanSchwinger operator associated with $E+V$. One easily checks that $E+V$ has its lowest eigenvalue 0 , see [7]. (This follows from the fact that $\hat{V} \leq 0$ is negative and so the ground state of $E+V$ can be chosen to have non-negative Fourier transform. Hence, it is not orthogonal to $\alpha_{\mu, V}$, which is an eigenfunction with eigenvalue 0 .) Thus, $B_{\Delta}$ has -1 as its lowest eigenvalue.

Proposition 5 In the limit $\mu \rightarrow 0$, the function $\Delta$ satisfies $\Delta(\sqrt{\mu})=o(\mu)$.

Proof Suppose for contradiction that $\frac{\Delta(\sqrt{\mu})}{\mu}$ does not vanish. That is, suppose that there is some subsequence with $\Delta(\sqrt{\mu})>B \mu$ for $\mu \rightarrow 0$ for some constant $B>0$. We use the decomposition

$$
B_{\Delta}=V^{1 / 2} \frac{1}{p^{2}}|V|^{1 / 2}+m_{\mu}(\Delta)\left|V^{1 / 2}\right\rangle\left\langle|V|^{1 / 2}\right|+A_{\Delta, \mu}
$$

By the assumptions on $V$, the spectrum of $V^{1 / 2} \frac{1}{p^{2}}|V|^{1 / 2}$ is contained in $(-1, \infty)$. We show that the remaining two terms in the decomposition above vanish in the limit $\mu \rightarrow 0$, and so that the spectrum of $B_{\Delta}$ approaches that of $V^{1 / 2} \frac{1}{p^{2}}|V|^{1 / 2}$. Since the latter has its lowest eigenvalue strictly larger than -1 , we get a contradiction.

For $m_{\mu}(\Delta)$, we use Proposition 4 above. The only term that does not immediately vanish in the limit $\mu \rightarrow 0$ is the term

$$
\frac{\sqrt{\mu}}{4 \pi^{2}} \int_{1}^{\infty} \frac{\sqrt{1+s}}{\sqrt{s^{2}+\left(\frac{\Delta(\sqrt{\mu})}{\mu}\right)^{2}}}-\frac{1}{\sqrt{1+s}} \mathrm{~d} s
$$

By splitting this integral according to $s<\frac{\Delta(\sqrt{\mu})}{\mu}$ and $s>\frac{\Delta(\sqrt{\mu})}{\mu}$, we see that this term may be bounded by $C \mu^{-1 / 2} \Delta(\sqrt{\mu}) \leq C \mu^{1 / 4}$ by Proposition 3 . Hence, this term indeed also vanishes.

For the kernel of $A_{\Delta, \mu}$, we use that $\left|\frac{\sin b}{b}-1\right| \leq C \min \left\{1, b^{2}\right\} \leq C b^{\gamma}$ for any $0 \leq \gamma \leq 2$ for the specific choice of $\gamma=\frac{1}{2}$. Then,

$$
\begin{aligned}
\left|A_{\Delta, \mu}(x, y)\right| \leq & C|V(x)|^{1 / 2}|V(y)|^{1 / 2}|x-y|^{1 / 2}\left[\int_{0}^{\sqrt{2 \mu}}\left|\frac{1}{E}-\frac{1}{p^{2}}\right| p^{5 / 2} \mathrm{~d} p\right. \\
& \left.+\int_{\sqrt{2 \mu}}^{\infty}\left|\frac{1}{E}-\frac{1}{p^{2}}\right| p^{5 / 2} \mathrm{~d} p\right] .
\end{aligned}
$$


For the first integral, we bound $E(p) \geq \Delta(p) \geq \Delta(\sqrt{\mu})-C \mu^{5 / 4} \geq B^{\prime} \mu$ for sufficiently small $\mu$ and some $B^{\prime}>0$ by Proposition 3 . Thus,

$$
\int_{0}^{\sqrt{2 \mu}}\left|\frac{1}{E}-\frac{1}{p^{2}}\right| p^{5 / 2} \mathrm{~d} p \leq \int_{0}^{\sqrt{2 \mu}} \frac{1}{B^{\prime} \mu}(2 \mu)^{5 / 4}+(2 \mu)^{1 / 4} \mathrm{~d} p \leq C \mu^{3 / 4} .
$$

We bound the second integral as follows. First, with the substitution $s=\frac{p^{2}-\mu}{\mu}$ we get

$$
\begin{aligned}
\int_{\sqrt{2 \mu}}^{\infty}\left|\frac{1}{E_{\Delta}}-\frac{1}{p^{2}}\right| p^{5 / 2} \mathrm{~d} p & =\frac{\mu^{3 / 4}}{2} \int_{1}^{\infty}\left|\frac{1+s}{\sqrt{s^{2}+\left(\frac{\Delta(\sqrt{\mu} \sqrt{1+s})}{\mu}\right)^{2}}}-1\right| \frac{1}{(1+s)^{1 / 4}} \mathrm{~d} s \\
& \leq \frac{\mu^{3 / 4}}{2} \int_{1}^{\infty} \frac{1}{s(1+s)^{1 / 4}}+\frac{\sqrt{s^{2}+\left(\frac{\Delta(\sqrt{\mu} \sqrt{1+s})}{\mu}\right)^{2}}-s}{s(1+s)^{1 / 4}} \mathrm{~d} s \\
& \leq C \mu^{3 / 4}+C \mu^{-1 / 4} \int_{1}^{\infty} \frac{\Delta(\sqrt{\mu} \sqrt{1+s})}{s(1+s)^{1 / 4}} \mathrm{~d} s \\
& \leq C \mu^{1 / 2},
\end{aligned}
$$

where we used that $|\Delta(p)| \leq C \mu^{3 / 4}$. The integral $\iint|V(x)||V(y)||x-y| \mathrm{d} x \mathrm{~d} y<\infty$ is finite by the assumptions on $V$. Thus, $\left\|A_{\Delta, \mu}\right\|_{2} \leq C \mu^{1 / 2}$ vanishes, and we get the desired contradiction. We conclude that $\Delta(\sqrt{\mu})=o(\mu)$.

Using this refined bound, $\Delta(\sqrt{\mu})=o(\mu)$, we may use a dominated convergence argument to show that

$$
\begin{aligned}
m_{\mu}(\Delta)= & \frac{\sqrt{\mu}}{4 \pi^{2}}\left[\int_{0}^{1} \frac{\sqrt{1-s}-1}{s}+\frac{\sqrt{1+s}-1}{s}-\frac{1}{\sqrt{1-s}}-\frac{1}{\sqrt{1+s}} \mathrm{~d} s\right. \\
& \left.+\int_{0}^{1} \frac{2}{\sqrt{s^{2}+\left(\frac{\Delta(\sqrt{\mu})}{\mu}\right)^{2}}} \mathrm{~d} s+\int_{1}^{\infty} \frac{\sqrt{1+s}}{s}-\frac{1}{\sqrt{1+s}} \mathrm{~d} s+o(1)\right] .
\end{aligned}
$$

These integrals can be computed (somewhat easily by hand). This is done in [7]. We conclude that

$$
m_{\mu}(\Delta)=\frac{\sqrt{\mu}}{2 \pi^{2}}\left(\log \frac{\mu}{\Delta(\sqrt{\mu})}-2+\log 8+o(1)\right)
$$

in the limit $\mu \rightarrow 0$, i.e. this shows the first equality in Lemma 1 . In particular, $m_{\mu}(\Delta) \gg \sqrt{\mu}$. Now, we are interested in bounding $A_{\Delta, \mu}$.

Proposition 6 The operator $A_{\Delta, \mu}$ vanishes in the following sense.

$$
\lim _{\mu \rightarrow 0} \frac{\left\|A_{\Delta, \mu}\right\|_{2}}{m_{\mu}(\Delta)}=0
$$


Proof The proof is similar as above, only we give a more refined bound on the kernel. We bound the $\frac{\sin b}{b}$ term by

$$
\begin{aligned}
\left|\frac{\sin |p||x-y|}{|p||x-y|}-1\right| \leq & C\left[p^{2} Z^{2} 1_{\{|x-y|<Z\}}+|p|^{1 / 2}|x-y|^{1 / 2} 1_{\{|x-y|>Z\}}\right] 1_{\left\{p^{2}<2 \mu\right\}} \\
& +C|p|^{1 / 2}|x-y|^{1 / 2} 1_{\left\{p^{2}>2 \mu\right\}} .
\end{aligned}
$$

where $Z>0$ is arbitrary, and the constant $C$ does not depend on $Z$. Then,

$$
\begin{aligned}
\left|A_{\Delta, \mu}(x, y)\right| \leq & C|V(x)|^{1 / 2}|V(y)|^{1 / 2}\left[Z^{2} \int_{0}^{\sqrt{2 \mu}}\left|\frac{1}{E_{\Delta}}-\frac{1}{p^{2}}\right| p^{4} \mathrm{~d} p\right. \\
& +|x-y|^{1 / 2} 1_{\{|x-y|>Z\}} \int_{0}^{\sqrt{2 \mu}}\left|\frac{1}{E_{\Delta}}-\frac{1}{p^{2}}\right| p^{5 / 2} \mathrm{~d} p \\
& \left.+|x-y|^{1 / 2} \int_{\sqrt{2 \mu}}^{\infty}\left|\frac{1}{E_{\Delta}}-\frac{1}{p^{2}}\right| p^{5 / 2} \mathrm{~d} p\right] .
\end{aligned}
$$

Now, the first and second integrals may be bounded by $m_{\mu}(\Delta) \mu$ and $m_{\mu}(\Delta) \mu^{1 / 4}$ as follows. For any $\gamma$, we may bound

$$
\int_{0}^{\sqrt{2 \mu}}\left|\frac{1}{E_{\Delta}}-\frac{1}{p^{2}}\right| p^{\gamma} \mathrm{d} p \leq \int_{0}^{\sqrt{2 \mu}}\left(\frac{1}{E_{\Delta}}-\frac{1}{p^{2}}\right) p^{\gamma}+2 p^{\gamma-2} \mathrm{~d} p \leq C m_{\mu}(\Delta) \mu^{\frac{\gamma-2}{2}}
$$

Similarly as before, the last integral may be bounded by $\mu^{1 / 2} \ll m_{\mu}(\Delta)$. Again, by the assumptions on $V$ it follows that $\iint|V(x)||V(y)||x-y| \mathrm{d} x \mathrm{~d} y<\infty$ is finite. Thus, we get $\lim _{\mu \rightarrow 0} \frac{\left\|A_{\Delta, \mu}\right\|_{2}}{m_{\mu}(\Delta)}=0$ as desired.

We may decompose

$$
\begin{aligned}
1+B_{\Delta}= & \left(1+V^{1 / 2} \frac{1}{p^{2}}|V|^{1 / 2}\right) \\
& \times\left(1+\frac{m_{\mu}(\Delta)}{1+V^{1 / 2} \frac{1}{p^{2}}|V|^{1 / 2}}\left(\left|V^{1 / 2}\right\rangle\left\langle|V|^{1 / 2}\right|+\frac{A_{\Delta, \mu}}{m_{\mu}(\Delta)}\right)\right) .
\end{aligned}
$$

Since -1 is an eigenvalue of $B_{\Delta}$, we get that -1 is an eigenvalue of

$$
\frac{m_{\mu}(\Delta)}{1+V^{1 / 2} \frac{1}{p^{2}}|V|^{1 / 2}}\left(\left|V^{1 / 2}\right\rangle\left\langle|V|^{1 / 2}\right|+\frac{A_{\Delta, \mu}}{m_{\mu}(\Delta)}\right)
$$


Proposition 6 gives that the term $\frac{A_{\Delta, \mu}}{m_{\mu}(\Delta)}$ vanishes in the limit $\mu \rightarrow 0$. The other term has rank one, and thus, we get

$$
\lim _{\mu \rightarrow 0} \frac{-1}{m_{\mu}(\Delta)}=\left\langle|V|^{1 / 2}\left|\frac{1}{1+V^{1 / 2} \frac{1}{p^{2}}|V|^{1 / 2}}\right| V^{1 / 2}\right\rangle=4 \pi a
$$

This is, apart from the (weaker) error term, the second equality in Lemma 1. We now show that the rate of convergence is indeed $o\left(\mu^{1 / 2}\right)$.

First, we improve on Proposition 6. Since $m_{\mu}(\Delta)$ is of order 1 in the limit $\mu \rightarrow 0$, we get for the third integral in the proof of Proposition 6 that

$$
\int_{\sqrt{2 \mu}}^{\infty}\left|\frac{1}{E_{\Delta}}-\frac{1}{p^{2}}\right| p^{5 / 2} \mathrm{~d} p \leq C \mu^{1 / 2} \ll \mu^{1 / 4}
$$

Hence, for any $Z>0$ and a constant $C$ that does not depend on $Z$ we get the bound

$$
\limsup _{\mu \rightarrow 0} \frac{\left\|A_{\Delta, \mu}\right\|_{2}}{\mu^{1 / 4}} \leq C\left(\iint_{\{|x-y|>Z\}}|V(x) \| V(y)||x-y| \mathrm{d} x \mathrm{~d} y\right)^{1 / 2} .
$$

By the assumptions on $V$, the integrand here is integrable and so taking $Z \rightarrow \infty$ we get that

$$
\lim _{\mu \rightarrow 0} \frac{\left\|A_{\Delta, \mu}\right\|_{2}}{\mu^{1 / 4}}=0 .
$$

Additionally, $A_{\Delta, \mu}$ vanishes in the limit $\mu \rightarrow 0$. Thus, the operator

$$
1+V^{1 / 2} \frac{1}{p^{2}}|V|^{1 / 2}+A_{\Delta, \mu}
$$

is invertible for small $\mu$ and so we may write

$$
\begin{aligned}
1+B_{\Delta}= & \left(1+V^{1 / 2} \frac{1}{p^{2}}|V|^{1 / 2}+A_{\Delta, \mu}\right) \\
& \times\left(1+\frac{m_{\mu}(\Delta)}{1+V^{1 / 2} \frac{1}{p^{2}}|V|^{1 / 2}+A_{\Delta, \mu}}\left|V^{1 / 2}\right\rangle\left\langle|V|^{1 / 2}\right|\right) .
\end{aligned}
$$

Since -1 is an eigenvalue of $B_{\Delta}$, we get that -1 is an eigenvalue of the latter operator. This has rank one and so we get that

$$
\frac{-1}{m_{\mu}(\Delta)}=\left\langle|V|^{1 / 2}\left|\frac{1}{1+V^{1 / 2} \frac{1}{p^{2}}|V|^{1 / 2}+A_{\Delta, \mu}}\right| V^{1 / 2}\right\rangle .
$$


We decompose the middle operator on the right hand side as

$$
\begin{aligned}
\frac{1}{1+} & V^{1 / 2} \frac{1}{p^{2}}|V|^{1 / 2}+A_{\Delta, \mu} \\
= & \frac{1}{1+V^{1 / 2} \frac{1}{p^{2}}|V|^{1 / 2}}-\frac{1}{1+V^{1 / 2} \frac{1}{p^{2}}|V|^{1 / 2}} A_{\Delta, \mu} \frac{1}{1+V^{1 / 2} \frac{1}{p^{2}}|V|^{1 / 2}} \\
& +\frac{1}{1+V^{1 / 2} \frac{1}{p^{2}}|V|^{1 / 2}} A_{\Delta, \mu} \frac{1}{1+V^{1 / 2} \frac{1}{p^{2}}|V|^{1 / 2}+A_{\Delta, \mu}} A_{\Delta, \mu} \frac{1}{1+V^{1 / 2} \frac{1}{p^{2}}|V|^{1 / 2}},
\end{aligned}
$$

which is perhaps most easily seen by writing the left hand side as a power series in $A_{\Delta, \mu}$. Plugging this into Eq. (3), we get $4 \pi a$ for the first term. The second term gives

$$
\left\langle f\left|\operatorname{sgn} V A_{\Delta, \mu}\right| f\right\rangle, \quad \text { with } \quad f=\frac{1}{1+V^{1 / 2} \frac{1}{p^{2}}|V|^{1 / 2}} V^{1 / 2} .
$$

This function $f$ is the same function $f$, which was studied in [6]. There, it was shown that this function satisfies $f(x)|V(x)|^{1 / 2}(1+|x|) \in L^{1}$.

The third term in the expansion above is $o\left(\mu^{1 / 2}\right)$ by Eq. (2). We show that the second term is $o\left(\mu^{1 / 2}\right)$ as well.

Proposition 7 In the limit $\mu \rightarrow 0$, we have $\left\langle f\left|\operatorname{sgn} V A_{\Delta, \mu}\right| f\right\rangle=o\left(\mu^{1 / 2}\right)$.

Proof This is similar to the bound on $A_{\Delta, \mu}$ above. We bound the kernel of $A_{\Delta, \mu}$ by

$$
\begin{aligned}
\left|A_{\Delta, \mu}(x, y)\right| \leq & C|V(x)|^{1 / 2}|V(y)|^{1 / 2}\left[Z^{2} \int_{0}^{\sqrt{2 \mu}}\left|\frac{1}{E_{\Delta}}-\frac{1}{p^{2}}\right| p^{4} \mathrm{~d} p\right. \\
& +|x-y| 1_{\{|x-y|>Z\}} \int_{0}^{\sqrt{2 \mu}}\left|\frac{1}{E_{\Delta}}-\frac{1}{p^{2}}\right| p^{3} \mathrm{~d} p \\
& \left.+|x-y|^{3 / 4} \int_{\sqrt{2 \mu}}^{\infty}\left|\frac{1}{E_{\Delta}}-\frac{1}{p^{2}}\right| p^{11 / 4} \mathrm{~d} p\right] .
\end{aligned}
$$

These integrals are bounded by $\mu, \mu^{1 / 2}$ and $\mu^{5 / 8}$, respectively, similarly as in Proposition 6. (Recall that $m_{\mu}(\Delta)$ is of order 1.) Thus,

$$
\begin{aligned}
& \limsup _{\mu \rightarrow 0} \frac{\left|\left\langle f\left|\operatorname{sgn} V A_{\Delta, \mu}\right| f\right\rangle\right|}{\mu^{1 / 2}} \\
& \quad \leq C \iint_{\{|x-y|>Z\}}|f(x)||V(x)|^{1 / 2}|x-y||f(y)||V(y)|^{1 / 2} \mathrm{~d} x \mathrm{~d} y .
\end{aligned}
$$

Since $f(x)|V(x)|^{1 / 2}(1+|x|) \in L^{1}$, we get the desired by taking $Z \rightarrow \infty$.

We conclude that

$$
m_{\mu}(\Delta)=\frac{-1}{4 \pi a}+o\left(\mu^{1 / 2}\right)
$$


This concludes the proof of the second equality in Lemma 1 and thus the proof of Theorem 1.

Acknowledgements Most of this work was done as part of the author's master's thesis. The author would like to thank Jan Philip Solovej for his supervision of this process.

Funding Open Access funding provided by Institute of Science and Technology (IST Austria)

\section{Compliance with ethical standards}

Conflict of interest The author declares that they have no conflict of interest.

Open Access This article is licensed under a Creative Commons Attribution 4.0 International License, which permits use, sharing, adaptation, distribution and reproduction in any medium or format, as long as you give appropriate credit to the original author(s) and the source, provide a link to the Creative Commons licence, and indicate if changes were made. The images or other third party material in this article are included in the article's Creative Commons licence, unless indicated otherwise in a credit line to the material. If material is not included in the article's Creative Commons licence and your intended use is not permitted by statutory regulation or exceeds the permitted use, you will need to obtain permission directly from the copyright holder. To view a copy of this licence, visit http://creativecommons.org/licenses/by/4.0/.

\section{References}

1. Bardeen, J., Cooper, L.N., Schrieffer, J.R.: Theory of superconductivity. Phys. Rev. 108, 5 (1957)

2. Bräunlich, G., Hainzl, C., Seiringer, R.: Translation-invariant quasi-free states for fermionic systems and the BCS approximation. Rev. Math. Phys. 26(07), 1450012 (2014). https://doi.org/10.1142/ S0129055X14500123

3. Frank, R., Hainzl, C., Naboko, S., Seiringer, R.: The critical temperature for the BCS equation at weak coupling. J. Geom. Anal. 17, 559-567 (2007). https://doi.org/10.1007/BF02937429

4. Gor'kov, L.P., Melik-Barkhudarov, T.K.: Contributions to the theory of superfluidity in an imperfect Fermi gas. Sov. Phys. JETP 13(5), 1018 (1961)

5. Hainzl, C., Hamza, E., Seiringer, R., Solovej, J.P.: The BCS functional for general pair interactions. Commun. Math. Phys. 281, 349-367 (2008). https://doi.org/10.1007/s00220-008-0489-2

6. Hainzl, C., Seiringer, R.: The BCS critical temperature for potentials with negative scattering length. Lett. Math. Phys. 84, 99-107 (2008)

7. Hainzl, C., Seiringer, R.: Critical temperature and energy gap for the BCS equation. Phys. Rev. B 77, 184517 (2008). https://doi.org/10.1103/PhysRevB.77.184517

8. Hainzl, C., Seiringer, R.: Spectral properties of the BCS gap equation of superfluidity. Math. Results Quantum Mech. (2008). https://doi.org/10.1142/9789812832382_0009

9. Hainzl, C., Seiringer, R.: The Bardeen-Cooper-Schrieffer functional of superconductivity and its mathematical properties. J. Math. Phys. 57(2), 021101 (2016). https://doi.org/10.1063/1.4941723

10. Leggett, A.J.: Diatomic molecules and Cooper pairs. In: Pekalski, A., Przystawa, J.A. (eds.) Modern Trends in the Theory of Condensed Matter, pp. 13-27. Springer, Berlin (1980)

11. Lieb, E.H., Loss, M.: Analysis. Graduate Studies in Mathematics, vol. 14, 2nd edn. American Mathematical Society, Providence (2001)

12. Nozières, P., Schmitt-Rink, S.: Bose condensation in an attractive fermion gas: from weak to strong coupling superconductivity. J. Low Temp. Phys. 59, 195-211 (1985)

Publisher's Note Springer Nature remains neutral with regard to jurisdictional claims in published maps and institutional affiliations. 